\title{
Transmission Electron Microscopy of Vertically Stacked ErAs-InAs Semimetal - Quantum Dot Nanocomposite Heterostructures Grown on GaAs(001) Substrates
}

\author{
Krishnamurthy Mahalingam ${ }^{1}$, Yuanchang Zhang ${ }^{1}$, Kurt Eyink $^{1}$, Joseph Peoples ${ }^{1}$, Brittany Urwin ${ }^{1}$, \\ Lawrence Grazulis ${ }^{1}$ and Madelyn Hill ${ }^{1}$. \\ 1. Nano-Electronic Materials Branch, Air Force Research Laboratory, Wright-Patterson Air Force Base, \\ $\mathrm{OH}$, United States.
}

Epitaxial thin films combining InAs quantum dots (QDs) with ErAs metallic nanoparticles (MNPs) are emerging device materials with the potential for wide ranging optoelectronic applications, which are based on coupling the plasmonic properties of MNPs with the absorption/emission characteristics of QDs. Several critical parameters influence the formation of these hybrid structures, including the MNPQD size/shape, their separation, placement, and relative orientation, all of which critically depend on the strain field due to the QDs. We have recently developed a growth procedure aimed at optimizing these parameters, wherein the hybrid structures are grown epitaxial on (001) GaAs substrates by molecular beam epitaxy. Specifically, following the growth of InAs QD and GaAs spacer layer, the ErAs MNP is achieved in two steps: (1) co-deposition of InAs and ErAs to trigger preferential NP nucleation over underlying QDs due to associated strain field and (2) subsequent evaporation of In to form ErAs MNPs.

In this submission we present transmission electron microscope (TEM) studies aimed at obtaining quantitative information on many of the parameters mentioned above. In particular, we have applied recent advancements in TEM based techniques for defect characterization, and the examination of composition and strain distribution in the GaAs spacer layer surrounding the MNP-QD region. These studies were performed on MNP/QD hybrid layers in which the Er deposition rate was systematically changed by adjusting the cell temperature from $1140 \mathrm{C}$ to $1185 \mathrm{C}$. The GaAs spacer layer thickness between the NP/QD was maintained at nominal value of $15 \mathrm{~nm}$ and NP/QD layer was separated by a GaAs layer of nominal thickness of $150 \mathrm{~nm}$. The samples were examined using aberration corrected high resolution TEM and Z-contrast imaging techniques. In addition compositional analysis of the constituent layers was performed by X-ray energy dispersion spectroscopy (XEDS).

Figure 1 is a bright-field image of the overall structure showing the layers grown at different Er effusion cell temperatures. The image on the right is a magnification of the region indicated by the green arrow showing good alignment between the NP and the underlying QD. Several regions in the image were further analysed to examine the spatial correlation between NPs and the QDs. However Fig.1 also shows the presence of stacking faults in NPs formed by the co-deposition, which is not typically observed in NPs grown by conventional methods (Fig. 2). Figure 3 shows XEDS maps obtained from regions surrounding the NP/QD layers which indicates the presence of a thin In layer beneath the NP, thereby indicating that the temperature for evaporation was not high enough to completely get rid of deposited In. A comparison of the HAADF image and Er map also shows the locations from where the stacking faults nucleate. More detailed high-resolution TEM studies showing the strain field around these defects and the spatial correlation between the QDs and MNPs will be presented [1].

\section{References:}

[1] The authors acknowledge funding and support from the Air Force Office of Scientific Research under LRIR No. 17RXCOR427, Program Manager: Dr. Gernot Pomrenke. 


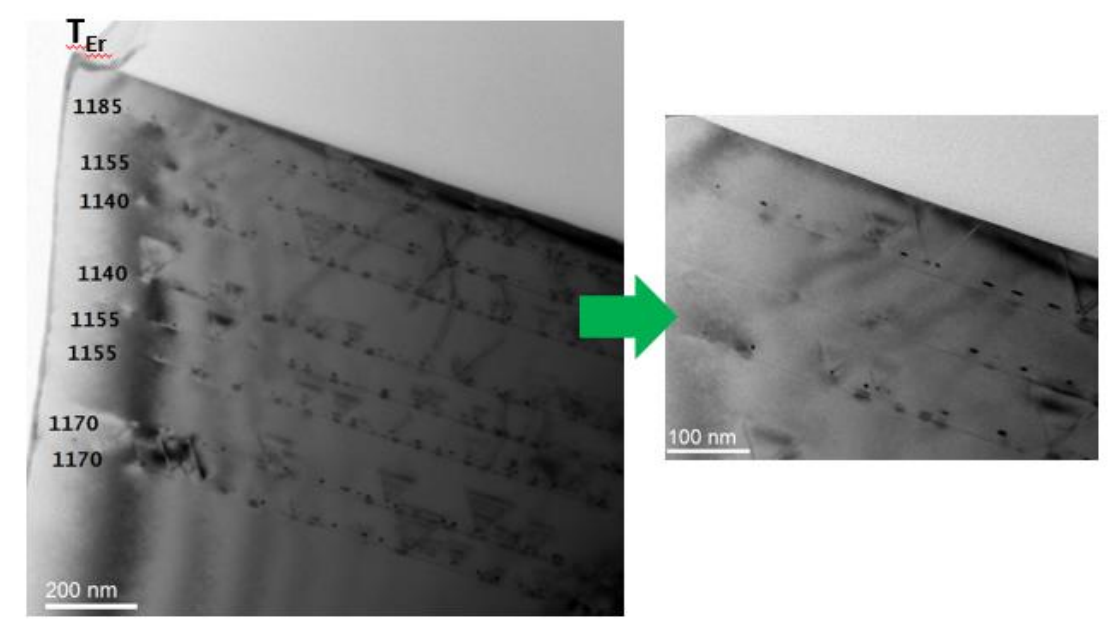

Figure 1. A TEM image of ErAs/InAs layers grown at different Er-cell temperatures as indicated. The image on right is a higher magnification of the top three layers showing good QD-NP spatial alignment.
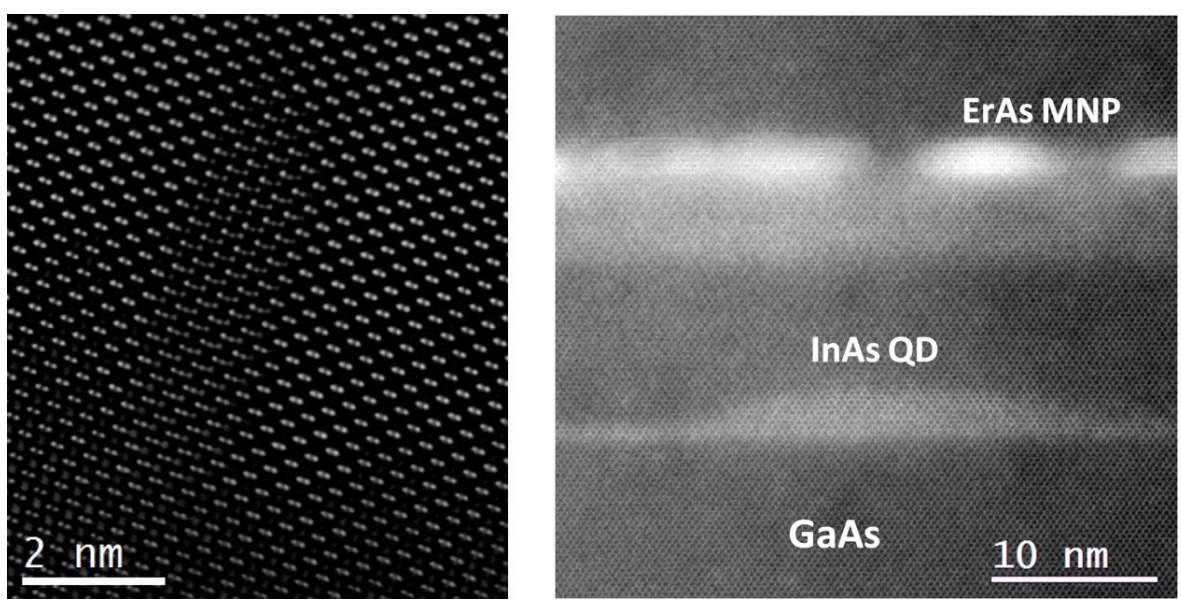

Figure 2. Left: Exit-wave reconstructed phase-image of an ErAs semimetal nanoparticle in GaAs, showing high structural quality of the embedded nanoparticles, wherein the rocksalt structure of the MNP and zinc-blende structure of GaAs are clearly resolved. Right: Z-contrast image of ErAs/InAs layers showing significant Er diffusion into the GaAs spacer layer.

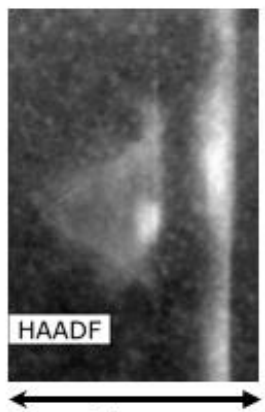

$45 \mathrm{~nm}$

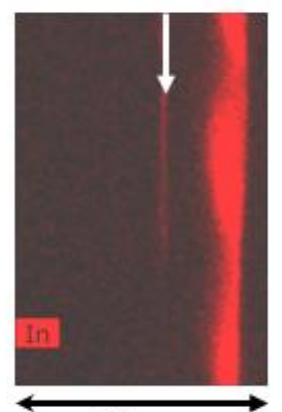

$45 \mathrm{~nm}$

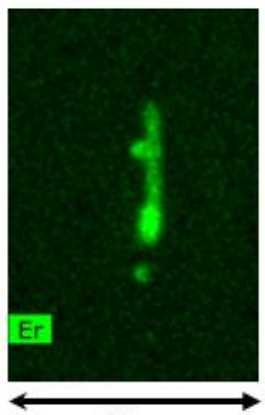

$45 \mathrm{~nm}$

Figure 3. HAADF-STEM image (left), XEDS-In map (middle) and XES-Er map (right) of a NP-QD layered structure. The white arrow in the middle image indicates presence of residual In beneath the NP. 